\title{
Increased expression of miR-155 and miR-222 is associated with lymph node positive status
}

\author{
Vladimir Chernyy, Vladimir Pustylnyak ${ }^{\bowtie}$, Vadim Kozlov, Lyudmila Gulyaeva \\ 1. The Institute of Molecular Biology and Biophysics, Novosibirsk, Timakova str., 2/12, Russia; \\ 2. Novosibirsk State University, Novosibirsk, Pirogova str., 1, Russia; \\ 3. Regional Clinical Oncological Hospital, Novosibirsk, Russia. \\ $\triangle$ Corresponding author: Vladimir O. Pustylnyak, PhD, Novosibirsk State University, Novosibirsk, Pirogova str., 1, Russia, 630090 E-mail: \\ pustylnyak@post.nsu.ru \\ (C) Ivyspring International Publisher. This is an open access article distributed under the terms of the Creative Commons Attribution (CC BY-NC) license \\ (https://creativecommons.org/licenses/by-nc/4.0/). See http://ivyspring.com/terms for full terms and conditions.
}

Received: 2017.08.01; Accepted: 2017.09.07; Published: 2018.01.01

\begin{abstract}
Identification of prognostic molecular markers of breast cancer is extremely important. The spreading out of the primary breast tumour cells to the lymphatic system is at the forefront of symbolising the first signs of distant organ metastasis. Deregulated genes in breast cancer tissues that spread to lymph nodes may show early predictive molecular markers. In the present study, we selected five microRNAs, which play a key function in the invasion-metastasis cascade. We investigated the levels of microRNAs in 80 paired samples of BC and matched adjoining tissues, and we examined the potential relationships between microRNA levels and positive lymph node status. Our results attest that three microRNAs (miR-21, miR-155, miR-222) were significantly up-regulated, whilst miR-205 was substantially down-regulated in $B C$ tissues in relation to normal adjoining tissues in a heterogeneous patient cohort. The high levels of two microRNAs, miR-155 and miR-222, showed a statistical relation with the positive lymph node status, especially in patients that had triple negative BC. Conversely, miR-155 was substantially down-regulated in tumour tissues of patients who received preoperative neoadjuvant chemotherapy (NAC) compared with tumour tissues of patients without NAC in cohorts sub-classified to lymph node positive status. Our findings show evidence that the miR-155 and the miR-222 can be defined as molecular markers in regards to cancer patients to prognosticate spread to the lymph node. They also showed that the miR-155 could have crucial significances in BC treatment.
\end{abstract}

Key words: breast cancer; lymph node metastasis; biomarker; microRNA.

\section{Introduction}

Tumour metastases often result in death of breast cancer (BC) patients [1]; however, our understanding of metastatic cellular processes is still controversial. In recent years, many studies have concentrated on establishing biomolecules that drive the metastatic process. The discovery of molecular biomarkers in the prognosis of tumour metastasis that can be applied to the routine practice of physicians is of crucial importance so as to powerfully control tumour progression. The metastatic cells that reach lymph nodes might come before the distant organ metastasis [2]. Deregulated genes in BC tissues that diffuse to the lymph nodes before forming distant organ metastases might present early biomarkers for patients with the risk of distant metastases.
In recent years, substantial success was achieved in the treatment and diagnosis of $B C$ due to the explosive development of modern molecular techniques. Numerous studies on BC have analysed different microRNAs that can influence and shape the cancer development and its progression [3]. MicroRNAs are small non-coding protein RNAs. It causes mRNA degradation and/or the blockade of mRNA translation [4]. MicroRNAs are involved in many physiological processes [5]. MicroRNAs can be both tumour suppressors and oncogenes [6]. High throughput assays have demonstrated that many microRNAs are deregulated in a majority of cancer [7, 8]. The microRNAs aberrant expression signatures have led to the proposal that they have prognostic, 
diagnostic, and therapeutic values. Expression of several microRNAs are altered in BC tissues when compared to healthy breast tissue or amongst different subtypes of BC $[9,10,11,12]$. Up- or down-regulation of several microRNAs in BC cells associated with tumour invasion and metastasis [13]. Some microRNAs have been involved in BC treatment response [14, 15].

It is known that primary $B C$ in patients with lymph node metastases may exhibit a greater invasive behaviour during the diagnosis period than do primary BC in patients without lymph node metastases. The differences might be due to deregulation of the expression of several regulatory microRNAs. Thus, in this study, the levels of tumour-suppressive microRNAs and oncogenic in BC tissues of patients that had or did not have a positive lymph node status were investigated. Previous reports literature search was conducted for the selection of microRNAs with a predictive potential in the invasion-metastasis cascade.

\section{Materials and Methods}

\section{Patients and tissue collection}

We received written consent from all patients. The research was given the greenlight to proceed by the Institute of Molecular Biology and Biophysics Ethics Committee in conformity with the 1975 Helsinki Declaration. All samples (pairs of the BC tissue and untransformed [adjoining normal] tissue samples) were obtained during surgery at the State Regional Oncology Centre (Novosibirsk) from 2011 to $2014(\mathrm{n}=80)$. Using liquid nitrogen, the tissue samples were frozen and kept at $-70{ }^{\circ} \mathrm{C}$. Clinico-pathological attributes of BC patients were recorded including age, occupation, ethnicity, menstrual and reproductive functions, clinical TNM stage, pathology diagnosis, molecular subtype and chemotherapy strategies (Table 1). The immunohisto-chemical streptavidin/peroxidise method was utilised to evaluate the hormonal receptor and HER2 status of the BC samples. All patient information was anonymised prior to analysis. Thirty-two patients received preoperative neoadjuvant chemotherapy (NAC) with 2 or 3 courses of FAC or CAF schemes of 5-fluorouracil, cyclophosphamide and doxorubicin. Surgery was performed 7-14 days after the end of NAC.

\section{Isolation of the total RNA, the cDNA synthesis and the real-time PCR}

The Vector-Best kit (Russia) was used to extract the total RNA from $50 \mathrm{mg}$ of frozen tissue while conforming to the producer's recommendations. The integrity of the extracted RNA was analysed by visualising the rRNA bands using gel electrophoresis, and the purity plus theconcentration of the total extracted RNA was ascertained by spectrophotometric method. For stem-loop RT-PCR [16], total RNA was reverse-transcribed by employing specific stem-loop primers using a SibEnzyme reagent kit (Russia). The estimated levels of microRNAs were measured by using real-time PCR with the SibEnzyme reagent kit (Russia) by employing the $\mathrm{CFX96^{ \textrm {TM } }}$ real-time PCR machine (Bio-Rad Laboratories, USA). The specific primers and probes were employed (sequences available on request to the authors). The relative changes of miRNA levels were calculated based on the PCR efficiency (E) and the $C_{t}$ and were normalised with U6 snRNA. U6 snRNA was used as a normalization internal control since it is mostly employed for this function in such studies of the relative levels of microRNAs [17]. The expression fold change for each one of the microRNA in the BC tissue versus paired adjacent (normal) tissue was computed by applying the formula that considers each of the PCR's amplification efficiency [18]. The PCR was executed in triplicate that included RT negative and no-template controls.

Table 1. Clinicopathological characteristics of BC patients.

\begin{tabular}{lc}
\hline Characteristic & Value, $\mathbf{n}(\mathbf{0})$ \\
\hline Total cases & $80(100 \%)$ \\
Age, median (range) & $58(40-80)$ \\
Menopausal State & \\
Premenopausal & $20(25 \%)$ \\
Postmenopausal & $60(75 \%)$ \\
ER status & \\
Positive & $58(72.5 \%)$ \\
Negative & $22(27.5 \%)$ \\
PR status & \\
Positive & $54(67.5 \%)$ \\
Negative & $26(32.5 \%)$ \\
Her2 status & \\
Positive & $18(22.5 \%)$ \\
Negative & $62(77.5 \%)$ \\
TNBC & \\
TNBC & $16(20 \%)$ \\
Non-TNBC & $64(80 \%)$ \\
Tumour size & \\
T1-2 & $70(87.5 \%)$ \\
T3-4 & $10(12.5 \%)$ \\
Lymph Node Involvement & $30(37.5 \%)$ \\
Yes & $50(62.5 \%)$ \\
No & \\
Neoadjuvant therapy & \\
Yes & \\
No & \\
\hline ER, estrogen receptor; PR, progesterone receptor; Her2, epidermal growth factor \\
receptor 2;TNBC, triple negative breast cancer. \\
\end{tabular}

\section{Statistical analysis}

A two-tailed Mann-Whitney test that was non-parametric was utilised to examine the 
differences in of the microRNAs expression in any two subgroups. A p-value $<0.05$ was seen as statistically substantial. MedCalc for Windows was employed for statistical analysis.

\section{Results}

We tested miRNA levels of 80 patients with BC. Five microRNAs were selected for analysis using RT-PCR. First, the levels of microRNAs were detected in all 80 pairs of tumor tissues and normal adjacent tissues normalized to U6 snRNA. We discovered that the levels of miR-21, miR-155, and miR-222 were substantially increased, whilst the levels of miR-205 were substantially decreased in BC tissues in comparison with their paired adjoining tissues (Table 2). We observed that miR-221 was slightly over-expressed in the $\mathrm{BC}$ tissue samples, but the difference in expression between $\mathrm{BC}$ tissue samples and normal adjoining tissue samples was not statistically significant (Table 2).

$\mathrm{BC}$ is extremely heterogeneous and it is very often assorted into various subtypes that are based on the hormonal receptors (ER, PR) expressionand HER2 [19]. Thus, we analysed the relationships that exist between microRNA expression and the molecular subtypes of BC. Tumours stained positive for ER in 58 patients $(72.5 \%)$, for PR in 54 patients $(67.5 \%)$, and for HER2 in 18 patients $(22.5 \%)$. Among these 80 patients, these are 16 cases $(20 \%)$ of breast cancer, which do not express the receptors (TNBC, Table 1). As shown in Table 3, hormone receptor statuses (ER and PR), HER2 status, as well as menopausal state were not correlated with microRNA expression.
However, the miR-205 expression levels were substantially lower $(p=0.003)$ in HER2-positive patients. Then, we analysed the expression of five microRNAs between TNBC and non-TNBC. We observed that only miR-21 was substantially over-expressed $(\mathrm{p}=0.026)$ in TNBC compared to non-TNBC (Table 3). The differences in expression levels of four other microRNAs were not statistically significant.

To identify microRNAs' predictive potential for metastasis, we investigated the levels of five microRNAs of BC tissues normalised to normal adjacent tissues in groups of patients who had or did not have lymph node status that was positive. In the heterogeneous cohort, thirty patients $(37.5 \%)$ had lymph node invasion and fifty patients did not have lymph node metastasis. The miR-155 and miR-222 levels were substantially up-regulated in BC tissues of lymph node metastasis patients in relation to $\mathrm{BC}$ tissues of patients without, while the differences in miR-21, miR-205, and miR-221 were not statistically significant (Table 3).

Table 2. The relative levels of miR-21, miR-155, miR-221, miR-222, and miR-205 in tissue samples from BC patients.

\begin{tabular}{llll}
\hline MicroRNA & Number of patients & Median $^{\mathrm{a}}($ Range $)$ & $\mathrm{p}$-Value \\
\hline miR-21 & 80 & $3.82(2.64-6.45)$ & $<0.0001$ \\
miR-155 & 80 & $6.57(3.26-9.74)$ & $<0.0001$ \\
miR-221 & 80 & $1.13(0.92-1.36)$ & 0.172 \\
miR-222 & 80 & $1.24(1.14-1.55)$ & $<0.001$ \\
miR-205 & 80 & $0.34(0.13-0.54)$ & $<0.0001$ \\
\hline
\end{tabular}

aMedian of relative change of miRNA levels in the breast tumour versus paired normal (adjoining) tissue.

Table 3. The association between the levels of miR-21, miR-155, miR-205, miR-221, and miR-222 in tissue samples from BC patients and different clinical and histopathology features.

\begin{tabular}{|c|c|c|c|c|c|c|c|c|c|c|c|}
\hline \multirow[t]{2}{*}{ Patient Presentation } & \multicolumn{11}{|c|}{ Relative Expression ${ }^{\mathrm{a}}$ of miRNAs and p-Value of different categories } \\
\hline & $\mathrm{N}$ & miR-21 & p-Value & miR-155 & $p$-Value & miR-205 & p-Value & miR-221 & $p$-Value & miR-222 & p-Value \\
\hline \multicolumn{12}{|l|}{ Menopausal State } \\
\hline Premenopausal & 20 & 5.93 & 0.112 & 5.24 & 0.942 & 0.37 & 0.555 & 1.31 & 0.605 & 1.72 & 0.516 \\
\hline Postmenopausal & 60 & 3.00 & & 7.94 & & 0.41 & & 1.02 & & 1.08 & \\
\hline \multicolumn{12}{|l|}{ ER status } \\
\hline Positive & 58 & 2.82 & 0.880 & 6.19 & 0.779 & 0.34 & 0.829 & 1.13 & 0.812 & 1.14 & 0.532 \\
\hline Negative & 22 & 4.25 & & 9.38 & & 0.42 & & 1.03 & & 1.22 & \\
\hline \multicolumn{12}{|l|}{ PR status } \\
\hline Positive & 54 & 3.38 & 0.805 & 7.94 & 0.309 & 0.29 & 0.355 & 1.44 & 0.377 & 1.27 & 0.116 \\
\hline Negative & 26 & 6.45 & & 3.68 & & 0.53 & & 1.13 & & 1.04 & \\
\hline \multicolumn{12}{|l|}{ Her2 status } \\
\hline Positive & 18 & 3.38 & 0.489 & 3.68 & 0.142 & 0.03 & 0.003 & 1.02 & 0.612 & 1.12 & 0.187 \\
\hline Negative & 62 & 4.28 & & 6.33 & & 0.63 & & 1.14 & & 1.25 & \\
\hline \multicolumn{12}{|l|}{ TNBC } \\
\hline TNBC & 16 & 9.00 & 0.026 & 9.54 & 0.709 & 0.63 & 0.500 & 1.36 & 0.258 & 1.77 & 0.387 \\
\hline Non-TNBC & 64 & 3.00 & & 5.71 & & 0.31 & & 1.05 & & 1.14 & \\
\hline \multicolumn{12}{|l|}{$\begin{array}{l}\text { Lymph Node } \\
\text { Involvement }\end{array}$} \\
\hline Yes & 30 & 4.25 & 0.265 & 8.05 & 0.004 & 0.42 & 0.795 & 1.11 & 0.419 & 1.52 & 0.005 \\
\hline No & 50 & 2.88 & & 2.44 & & 0.40 & & 1.18 & & 1.04 & \\
\hline
\end{tabular}

a Median of relative change of miRNA levels in the breast tumour versus paired normal (adjacent) tissue.

ER, estrogen receptor; PR, progesterone receptor; Her2, epidermal growth factor receptor 2; TNBC, triple-negative breast cancer. 
We additionally examined the association of two microRNA levels, the miR-155 and the miR-222, in clinical sub cohorts classified by lymph node positive status and molecular subtypes of BC. No major correlations was observed between these two microRNAs' expressions and hormone receptor as well as HER2 status in clinical cohorts sub-classified to lymph node positive status (Table 4). However, we discovered that both the miR-155 ( $p=0.019)$ and the miR-222 $(p=0.048)$ were substantially over-expressed in TNBC in comparison with non-TNBC lymph node metastasis patients (Table 4).

$B C$ patients that have lymph node metastases are often treated with neoadjuvant chemotherapy than patients who do not have detectable metastases. Twenty patients in our cohort sub-divided to lymph node-positive $(66.6 \%)$ received preoperative neoadjuvant chemotherapy (NAC) with 2 or 3 courses of FAC or CAF schemes of 5-fluorouracil, cyclophosphamide and doxorubicin. When the lymph node positive status-associated microRNA levels were compared, miR-155 ( $\mathrm{p}=0.007)$ was down-regulated in tumour tissues of patients who received NAC compared with tumour tissues of patients without NAC, while the differences in miR-222 were not statistically significant (Table 4).

Table 4. Relative levels of miR-155 and miR-222 in BC tissues from patients with positive lymph node status of different clinical and histopatology presentations.

\begin{tabular}{lccccc}
\hline Patient Presentation & \multicolumn{5}{c}{$\begin{array}{l}\text { Relative Expression } \\
\text { different categories }\end{array}$} \\
\cline { 2 - 6 } & $\mathrm{N}$ & miR-155 & p-Value & miR-222 & $p$-Value \\
\hline ER status & 19 & 2.54 & 0.599 & 1.13 & 0.237 \\
Positive & 11 & 4.74 & & 1.41 & \\
Negative & 17 & 2.64 & 0.575 & 1.21 & 0.313 \\
PR status & 13 & 2.44 & & 1.13 & \\
Positive & & & & & \\
Negative & 14 & 2.84 & 0.114 & 1.13 & 0.628 \\
Her2 status & 16 & 6.28 & & 1.10 & \\
Positive & & & & & \\
Negative & 8 & 11.48 & 0.019 & 1.55 & 0.048 \\
TNBC & 22 & 3.44 & & 1.03 & \\
TNBC & & & & & \\
Non-TNBC & 20 & 3.65 & 0.037 & 1.42 & 0.204 \\
Neoadjuvant therapy & & & & & \\
Yes & 10 & 8.79 & & 1.24 & \\
No &
\end{tabular}

aMedian of relative change of miRNA levels in the breast tumour versus paired normal (adjacent) tissue.

ER, estrogen receptor; PR, progesterone receptor; Her2, epidermal growth factor receptor 2; TNBC, triple-negative breast cancer.

\section{Discussion}

MicroRNAs show great potential for BC treating, although their use in routine clinical practise is yet a long-term objective [13]. The hunt for BC prognostic biomarkers is an extremely topical key work. Metastasis is one of the critical causes of BC patient's death. The spread of primary BC cells to the lymph nodes is at the forefront of symbolising the first signs of metastatic expansion [2]. Thus, genes that have an increased or decreased expression levels in BC tissues in patients with lymph node metastases may represent early biomarkers of the risk of developing distant metastases. In this study, we determined the levels of tumour-suppressive and oncogenic microRNAs in paired samples of $\mathrm{BC}$ and adjoining normal tissues and investigated the potential relationships between microRNAs levels and lymph node metastasis. We selected five microRNAs that are involved in the tumour invasion-metastasis cascade. In accordance with previous studies, our results demonstrated that the levels of miR-21, miR-155, and miR-222 were increased in $B C$ tissue in relation to adjoining normal tissue, whereas miR-205 levels were decreased in our cohort of $\mathrm{BC}$ patients. The difference in miR-221 expression in the BC tissue samples and the adjoining tissue samples was not statistically substantial. Our current data are in agreement with former studies, which indicated that the miR-21, the miR-155 and the miR-222 are oncogenes, while the miR-205 is a tumor suppressor. It can be suggested that these microRNAs have a general purpose in the tumorigenic procedure in all BC subtypes.

MiR-21 is a well-studied biomarkers associated with poor prognosis. Recently, the miR-21 high levels have been demonstrated in many different cancer types, including BC, in which miR-21 is needed both, in modulating cell rapid multiplication and metastasis by modulating multiple target genes [20, 21]. MiR-155 is also found to be over-expressed in a variety of cancer. It was shown that up-regulated levels of miR-155 are consociated with lymph node metastasis [22]. Levels of miR-205 are down-regulated in BC tissue samples in relation to adjoining tissue and are consociated with negative regulation of the epithelial-to-mesenchymal transition (EMT), which was discovered to act a critical function in a tumour invasion-metastasis cascade [23]. Both the miR-221 and the miR-222 inhibit TRPS1 gene, which inhibits EMT through a decrease in the level of ZEB2 [24]. Thus, an increase in the levels of both microRNAs might lead to a rise in cell migration and invasion through the regulation of EMT.

No significant correlation between microRNA levels and hormonal receptor status was detected. At the same time, by statistical analysis, we discovered that the relative miR-205 expression in HER2-positive tumours was substantially lower in relation to HER2-negative tumours. Previously, it was demonstrated that miR-205 directly influences the level of HER3 [25], which acts a crucial and essential function in HER2-mediated BC development and progression by the PI3K/Akt pathway activation [26]. 
Additionally, we noted that only one of the oncogenic microRNAs, miR-21, was over-expressed in TNBC, a highly aggressive $\mathrm{BC}$ phenotype, compared to non-TNBC. Concerning miR-21 in TNBC, our findings were consistent with other studies $[27,28]$.

Our results reveal that two oncogenic microRNAs, miR-155 and miR-222, were highly expressed in BC tissue of lymph node metastasis patients. This data signalled that miR-155 and miR-222 might play critical roles in BC progression and metastasis. A possible statistical relation in high miR-21 levels and lymph node metastasis BC patients was reported [29], whereas in our tumour cohort, miR-21 expression levels did not significantly make a causal connection with lymph node status. Additionally, it was antecedently depicted that up-regulated miR-221 and miR-222 levels are linked with metastases formation in BC patients [30]. In our tumour cohort, we demonstrated the association between miR-222 levels and lymph node status. However, no association could be identified between miR-221 levels and lymph node status.

Previously, miR-221 has been shown to exhibit an ambiguous pattern of expression, majorly expressed in some BC sample tissues while entirely absent in others [31]. Moreover, it was suggested that levels of miR-221 depend on the tumor type and cellular content [32]. Down-regulation of miR-205 in TNBC patients was mutually related with lymph node metastasis [33]. Our data revealed a decrease in miR-205 expression levels in tumour tissues versus paired adjoining normal tissues. Further to that, there was no variation in the levels of miR-205 identified in positive and negative lymph node status cancer groups.

It was shown that molecular subtype of BC, based on the expression of hormonal receptors and HER2, is useful in forecasting the risk of a lymph node metastasis [34]. Two microRNAs, which were demonstrated to be enhanced in $\mathrm{BC}$ patients that had lymph node metastases relative to those $B C$ patients without lymph node metastases, were selected for further analysis. We investigated the link between the miR-155 expression and miR-222 expression in clinical cohorts sub-divided to lymph node positive status and molecular subtypes of BC. No significant correlation between both microRNAs' expression levels and hormonal receptor and HER2 status was recorded, whereas both microRNAs expression levels were substantially over-expressed in TNBC in relation to non-TNBC in patients that have lymph node metastasis.

Both miR-155 and miR-222 participate in EMT regulation and the expression of these miRNAs leads to the induction of tumour invasion-metastasis cascade $[30,35,36]$. The data proposed that miR-155 and miR-222 might be effective biomarkers for BC with invasive capabilities in the clinical stratification of BC, especially TNBC. TNBC displays an extremely aggressive tumour phenotype and a worse prognosis in comparison with other BC subtypes. Thus, TNBC patients have an increased likelihood of lymph node and distant organ metastasis. Additionally, high risk of progressive TNBC is often linked to inactivation of the tumour suppressor BRCA1 [37]. It has been shown that BRCA1 reduces the expression of miR-155 through the recruitment of histone deacetylase to the miR-155 promoter [38].

Conversely, several studies suggested a protective function of miR-155 in BC, especially in TNBC [39]. Further study has shown that miR-155 directly binds to the RAD51 mRNA and thereby reduced the protein level of RAD51 [40]. RAD51 participates in the homologous recombination, which is a fundamental pathway for the repair of DNA breaks produced by chemotherapeutic agents or ionising radiation [40, 41]. Thus, over-expression of miR-155 reducesthe efficiency of DNA double-strand breaks repair and increases sensitivity to therapy, which forms the killing lesion [42]. Apparently, miR-155 might exhibit dual purposes that are founded on cellular environment.

BC patients with positive lymph node status are often treated using preoperative neoadjuvant chemotherapy (NAC) than patients who do not have detectable metastases. The impact of neoadjuvant chemotherapy on the biology of $\mathrm{BC}$ cells is still controversial. It is well known that chemical compounds, including chemotherapeutic agents [43, 44, and 45], can regulate the expression of microRNAs. Few studies have investigated the consequences that chemotherapy has on miRNA expression profiles. Although these profiles were discovered, the mechanistic summary and the entire potential of microRNAs is yet not realised. In the present study, when the lymph node positive status associated microRNA levels were compared, we discovered that the miR-155 levels were substantially down-regulated in $\mathrm{BC}$ tissues of patients who received NAC compared to $B C$ tissues of patients without NAC. These results agree with a recent study that examined alternations in gene expression in tissue samples of BC patients treated using neoadjuvant chemotherapy [46]. Microarray analysis of $B C$ tissues revealed genes that were significantly deregulated after NAC. RAD51 gene expression was up-regulated few hours after initiation of NAC. Up-regulation of RAD51 may be driven by lower expression levels of miR-155. It could be speculated that despite initial sensitivity to chemotherapy, 
metastatic relapse seems to occur at higher rates, especially for TNBC [47], by the decrease of miR-155 and the increase of RAD51 by NAC. Large, well-planned research studies should be carried out to affirm our hypothesis.

Thus, our data demonstrate that the miR-155 and miR-222 levels are higher in BC tissues in relation to those in matched adjacent tissues and correlated with lymph node metastasis. These microRNAs might act a part in BC progression, and detecting their cellular levels should be further evaluated as prognostic biomarkers for predicting the lymph node metastasis of BC, especially in TNBC patients. Better understanding of miR-155 and miR-222 can be specifically useful in the betterment for $\mathrm{BC}$ prognosis and treatment.

\section{Acknowledgements}

This research study was backed by the Russian Science Foundation (project No. 15-15-30012).

\section{Competing Interests}

The authors have declared that no competing interest exists.

\section{References}

1. Redig AJ, McAllister SS. Breast cancer as a systemic disease: a view of metastasis. J Intern Med. 2013; 274: 113-126.

2. Eccles S, Paon L, Sleeman J. Lymphatic metastasis in breast cancer: importance and new insights into cellular and molecular mechanisms. ClinExp Metastasis. 2007; 24: 619-636.

3. Tang I, Ahmad A, Sarkar FH. The Role of MicroRNAs in Breast Cancer Migration, Invasion and Metastasis. International Journal of Molecular Sciences.2012; 13: 13414-13437.

4. Bartel DP. MicroRNAs:genomics, biogenesis, mechanism, and function. Cell 2004; 116:281-297.

5. Esquela-Kerscher A, Slack FJ. Oncomirs - microRNAs with a role in cancer. Nat Rev Cancer. 2006; 6: 259-269.

6. Croce CM. Causes and consequences of microRNA dysregulation in cancer. Nat Rev Genet. 2009; 10: 704-714

7. Lu J, Getz G, Miska EA, et al. MicroRNA expression profiles classify human cancers. Nature. 2005; 435: 834-838.

8. Volinia S, Calin GA, Liu CG, et al. A microRNA expression signature of human solid tumors defines cancer gene targets. ProcNatlAcadSci U S A. 2006; 103: 2257-2261.

9. Blenkiron LD, Goldstein NP, Thorne I, et al. MicroRNA expression profiling of human breast cancer identifies new markers of tumor subtype. Genome Biol. 2007; 8: R214

10. Mattie MD, Benz CC, Bowers J, et al. Optimized high-throughput microRNA expression profiling provides novel biomarker assessment of clinical prostate and breast cancer biopsies. Mol Cancer. 2006; 5: 24 .

11. Lowery AJ, Miller N, Devaney A, et al. MicroRNA signatures predict oestrogen receptor, progesterone receptor and HER2/neu receptor status in breast cancer. Breast Cancer Res. 2009; 11: R27.

12. Dai $X, C$ Chen $A, B a i Z$. Integrative investigation on breast cancer in ER, PR and HER2-defined subgroups using mRNA and miRNA expression profiling. Sci Rep. 2014; 4: 6566.

13. Amorim M, Salta S, Henrique R, et al. Decoding the usefulness of non-coding RNAs as breast cancer markers. J Transl Med. 2016; 14: 265.

14. Ohzawa H, Miki A, Teratani T, et al. Usefulness of miRNA profiles for predicting pathological responses to neoadjuvant chemotherapy in patients with human epidermal growth factor receptor2-positive breast cancer. OncolLett. 2017; 13: 1731-1740.

15. Raychaudhuri M, Bronger $\mathrm{H}$, Buchner $\mathrm{T}$, et al. MicroRNAs miR-7 and miR-340 predict response to neoadjuvant chemotherapy in breast cancer. Breast Cancer Res Treat.2017; 162: 511-521.

16. Chen C, Ridzon DA, Broomer AJ, et al. Real-time quantification of microRNAs by stem-loop RT-PCR. Nucleic Acids Res. 2005; 33: e179.

17. Schwarzenbach H, da Silva AM, Calin G, et al. Data Normalization Strategies for MicroRNA Quantification. Clin Chem. 2015; 61: 1333-1342.
18. Pfaffl MW. A new mathematical model for relative quantification in real-time RT-PCR. Nucleic Acids Res. 2001; 29: e45.

19. Harris L, Fritsche H, Mennel R, et al. American Society of Clinical Oncology 2007 update of recommendations for the use of tumor markers in breast cancer. J Clin Oncol. 2007; 25: 5287-5312.

20. Song B, Wang C, Liu J, et al. MicroRNA-21 regulates breast cancer invasion partly by targeting tissue inhibitor of metalloproteinase 3 expression. J Exp Clin Cancer Res. 2010; 29: 29.

21. Zhang C, Liu K, Li T, et al. miR-21: A gene of dual regulation in breast cancer. Int J Oncol.2016; 48: 161-172.

22. Chen J, Wang B-C, Tang J-H. Clinical significance of MicoRNA-155 expression in human breast cancer. J Surg Oncol. 2012; 106: 260-266.

23. Gregory PA, Bert AG, Paterson EL, et al. The miR-200 family and miR-205 regulate epithelial to mesenchymal transition by targeting ZEB1 and SIP1. Nat Cell Biol. 2008; 10: 593-601.

24. Stinson S, Lackner MR, Adai AT, et al. TRPS1 targeting by miR-221/222 promotes the epithelial-to-mesenchymal transition in breast cancer. Sci Signal. 2011; 4: ra41.

25. Iorio MV, Casalini P, Piovan C, et al. MicroRNA-205 regulates HER3 in human breast cancer. Cancer Res. 2009; 69: 2195-2200.

26. Stern DF. ERBB3/HER3 and ERBB2/HER2 duet in mammary development and breast cancer. J Mammary Gland Biol Neoplasia. 2008; 13: 215-223.

27. Radojicic J, Zaravinos A, Vrekoussis T, et al. MicroRNA expression analysis in triple-negative (ER, PR and Her2/neu) breast cancer. Cell Cycle. 2011; 10(3): 507-517.

28. Thakur S, Grover RK, Gupta S, et al. Identification of Specific miRNA Signature in Paired Sera and Tissue Samples of Indian Women with Triple Negative Breast Cancer. PLoS ONE. 2016; 11: e0158946.

29. Rask L, Balslev E, Søkilde R, et al. Differential expression of miR-139, miR-486 and miR-21 in breast cancer patients sub-classified according to lymph node status. Cell Oncol (Dordr). 2014; 37: 215-227.

30. Falkenberg N, Anastasov N, Rappl K, et al. MiR-221/-222 differentiate prognostic groups in advanced breast cancers and influence cell invasion. $\mathrm{Br} \mathrm{J}$ Cancer. 2013; 109: 2714-2723.

31. Sempere LF, Christensen M, Silahtaroglu A, et al. Altered MicroRNA expression confined to specific epithelial cell subpopulations in breast cancer. Cancer Res. 2007; 67: 11612-11620.

32. Volinia S, Galasso M, Sana ME, et al. Breast cancer signatures for invasiveness and prognosis defined by deep sequencing of microRNA. Proc Natl Acad Sci U S A. 2012; 109: 3024-3029.

33. Berber U, Yilmaz I, Narli G, et al. miR-205 and miR-200c: Predictive Micro RNAs for Lymph Node Metastasis in Triple Negative Breast Cancer. J Breast Cancer. 2014; 17: 143-148

34. Crabb SJ, Cheang MC, Leung S, et al. Basal breast cancer molecular subtype predicts for lower incidence of axillary lymph node metastases in primary breast cancer. Clin Breast Cancer. 2008; 8: 249-256.

35. Kong W, Yang H, He L, et al. MicroRNA-155 is regulated by the transforming growth factor beta/Smad pathway and contributes to epithelial cell plasticity by targeting RhoA. Mol Cell Biol. 2008; 28: 6773-6784.

36. Mattiske S, Suetani RJ, Neilsen PM, et al. The oncogenic role of miR-155 in breast cancer. Cancer Epidemiol Biomarkers Prev. 2012; 21: 1236-1243.

37. Podo F, Buydens LM, Degani H, et al. Triple-negative breast cancer: present challenges and new perspectives. Mol Oncol. 2010; 4: 209-229.

38. Chang S, Wang RH, Akagi K, et al. Tumor suppressor BRCA1 epigenetically controls oncogenic microRNA-155. Nat Med. 2011; 17: 1275-1282.

39. Cascione L, Gasparini P, Lovat F, et al. Integrated microRNA and mRNA signatures associated withsurvival in triple negative breast cancer. PLoS One. 2013; 8: e55910.

40. Arnaudeau C, Lundin C, Helleday T. DNA double-strand breaks associated with replication forks are predominantly repaired by homologous recombination involving an exchange mechanism in mammalian cells. J Mol Biol. 2001; 307: 1235-1245.

41. Baumann P, Benson FE, West SC. Human Rad51 protein promotes ATP-dependent homologous pairing and strand transfer reactions in vitro. Cell. 1996; 87: 757-766.

42. Gasparini P, Lovat F, Fassan M, et al. Protective role of miR-155 in breast cancer through RAD51 targeting impairs homologous recombination after irradiation. Proc Natl Acad Sci U S A. 2014; 111: 4536-4541.

43. Kovalchuk O, Filkowski J, Meservy J, et al. Involvement of microRNA-451 in resistance of the MCF-7 breast cancer cells to chemotherapeutic drug doxorubicin. Mol Cancer Ther.2008; 7: 2152-2159.

44. Tormo E, Pineda B, Serna E, et al. MicroRNA Profile in Response to Doxorubicin Treatment in Breast Cancer. J Cell Biochem. 2015; 116: 2061-2073.

45. Al-Khanbashi M, Caramuta S, Alajmi AM, et al. Tissue and Serum miRNA Profile in Locally Advanced Breast Cancer (LABC) in Response to Neo-Adjuvant Chemotherapy (NAC) Treatment. PLoS One. 2016; 11: e0152032.

46. Magbanua MJ, Wolf DM, Yau C, et al. Serial expression analysis of breast tumors during neoadjuvant chemotherapy reveals changes in cell cycle and immune pathways associated with recurrence and response. Breast Cancer Res. 2015; 17: 73.

47. Carey LA, Dees EC, Sawyer L, et al. The triple negative paradox: primary tumor chemosensitivity of breast cancer subtypes. Clin Cancer Res. 2007; 13: 2329-2334. 